\title{
Experimental Testing and Model Validation for Ocean Wave Energy Harvesting Buoys
}

\author{
Douglas A. Gemme ${ }^{1}$, Steven P. Bastien ${ }^{1}$, Raymond B. Sepe, Jr. ${ }^{1}$, John Montgomery ${ }^{2}$, Stephan T. Grilli ${ }^{2}$, Annette Grilli ${ }^{2}$ \\ ${ }^{1}$ Electro Standards Laboratories \\ 36 Western Industrial Drive \\ Cranston, RI 02921, USA \\ ${ }^{2}$ University of Rhode Island \\ Department of Ocean Engineering \\ Narragansett, RI 02882, USA
}

\begin{abstract}
Methodology and results are presented for numerical simulations and field experiments using point absorption ocean wave energy harvesting buoy systems, using the heave motion of the buoys to produce useful electrical power. Two approaches, a direct-drive system and a resonantdrive system are analyzed. These systems are not designed for large scale grid power applications, but rather for relatively low-power ocean sensor and communications applications, with power requirements in the 1-10 W range. The field experiments provided useful data for model verification and validation purposes. Results showed that RMS values for armature displacement and armature velocity and mean harvested electrical power were generally within $10 \%$ between model simulations and experimental data.
\end{abstract}

Index Terms - energy conversion, wave energy harvesting, linear generator, ocean energy, heaving buoy

\section{NOMENCLATURE}

A rigid-drive-system is an energy harvesting system which forces the linear generator armature to perfectly track (or mirror) the ocean wave surface elevation without amplification or attenuation of amplitude.

A direct-drive-system is an energy harvesting system which attempts to force the linear generator armature to track (or mirror) the ocean wave surface elevation, but is unable to do so perfectly, resulting in attenuation of the amplitude.

A resonant-drive-system is an energy harvesting system which allows the linear generator armature and/or the buoy to resonate with, and amplify, the ocean wave surface elevation.

\section{INTRODUCTION}

Ocean wave energy harvesting systems, designed for sensor buoys, convert wave motion into electricity, to allow operation under all weather conditions, while enabling enhanced functionality, higher performance and continuous operation. Such systems generate and accumulate energy that can be used to indefinitely power remote buoys, equipped with sensors arrays, as well as electronics for processing and communications. These power sources can be integrated with buoy systems to minimize the size of batteries, or eliminate the need for batteries if supercapacitors are used. Recently, a significant amount of work has been performed on the design, numerical modeling and field testing of buoy systems to be used as point absorbers to harvest energy from ocean waves [1-5]. A majority of these wave energy converters (WEC) target large amounts of power for grid applications. This paper, however, will focus on relatively low-power, distributed, point absorption WEC's and is an extension of preliminary design and numerical modeling work on direct-drive and resonant-drive systems aimed at harvesting power in the 1-10 W range in small sea states for sensor and communication applications [6]. An important contribution of this work was the development of accurate wave-to-wire simulation models that enable optimization of the various energy harvesting systems, while including important sub-system interactions. The theoretical system equations are structured as a nonlinear state-space model with a finite number of states, which lends itself to efficient numerical techniques, and is well suited to simulation using the well-established Simulink tool, which allows reliable and fast implementation of coupled and complicated nonlinear state-space system equations. Laboratory and field testing of 1:10 and 1:4 scale model prototypes of the two systems have been performed with reasonable results [7]. The following sections will provide an overview of the two types of systems developed, the numerical model developed to simulate these systems and results from field experiments of the systems as well as attempts to validate the model with the experimental results.

\section{SYSTEM OVERVIEW}

Two types of ocean wave energy harvesting systems have been developed; a direct-drive and a resonant-drive system. The direct-drive system consists of a linear electric generator (LEG), which is mounted vertically and directly driven by the wave induced heave motion of a surface float. It attempts to force the linear generator armature to move exactly with the sea surface elevation, but unlike a rigid- 
drive system, it is unable to track the motion perfectly, which results in differences between the buoy response and the sea surface elevation. The resonant-drive system uses a similar LEG, although it is driven by the motion of an inertial mass and resonant tuning of the system, which should amplify the sea surface elevation. In both systems, the LEG consists of a permanent magnet slider (armature), which is suspended by a spring system and allowed to oscillate within a two-phase coil stator. Electricity is produced by the coupled oscillations through the wave induced buoy motion. The challenge in designing most ocean energy harvesting systems stems from the highly coupled interaction of the sea state and the many components/subsystems, which are all individually difficult to analyze, even when isolated. Fig. 1 provides a block diagram showing the interactions. The randomness and complexity of ocean waves is one issue, and a useful system should operate over a wide range of conditions. While tools exist to describe ocean waves under linearized theory, and standard semi-empirical wave energy spectra are available as models, there is large coupling between the hydrodynamics and the buoy system, since significant energy is either extracted, dissipated or radiated by the buoy system. There are standard methods and models solving wave-buoy interactions (including incident, diffracted and radiated fields) when assuming small displacements and no viscous dissipations. The latter, which are important for small systems, introduce motion dependent nonlinear effects. Further, the LEG mechanically interacts with the buoy, and the force resulting is strong since it is directly related to energy generation. Also, the prediction of electrical performance of the LEG, which interacts with the mechanical dynamics of the LEG armature, requires electromagnetic calculations, and the LEG drives an energycapturing load, which is a controlled charging system in which the optimum parameters for the control algorithm depend on the sea-state. In order to deal with this complexity, it is necessary to analyze the concept of a rigiddrive system, which is difficult to achieve in practice. However, the performance results of the rigid-drive system prove useful in this discussion to provide of benchmark to determine the suitability of any real-world direct-drive or resonant-drive design. The analysis of these systems, including relevant equations, will be discussed in the following section on the numerical model which was developed for this work.

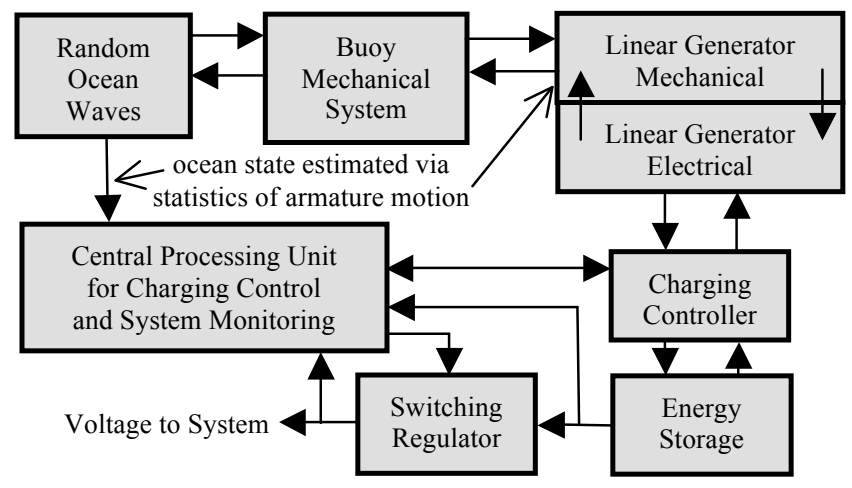

Figure 1. Block diagram of system interactions

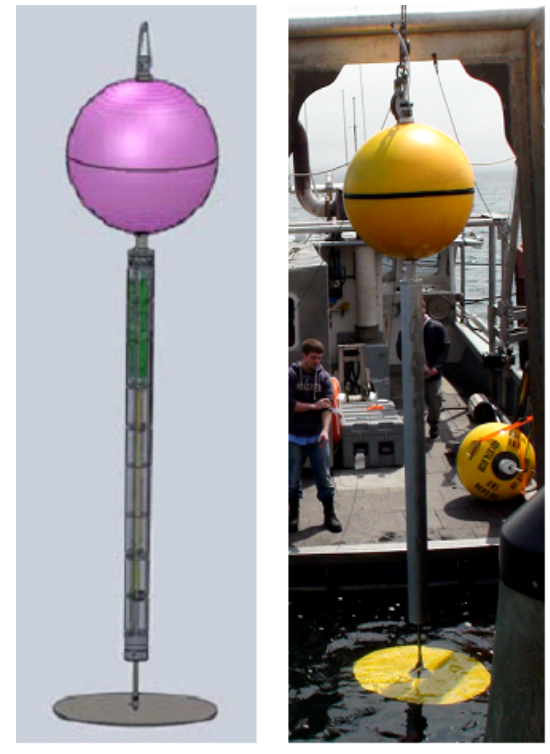

Figure 2. DC2 wave energy buoy; conceptual drawing (left) and 1:4 scale prototype (right)

The direct-drive system that was developed is referred to as the DC2 (Fig. 2). It consists of a spherical float which is rigidly attached to a watertight cylindrical canister, inside which the LEG is rigidly mounted. The permanent magnet armature of the LEG is then connected to a rod that exits through the bottom of the canister and is attached to a large resistance plate through a universal joint. The motion of the armature is constrained by a restoring spring attached at the top of the canister and by a bottom force produced from the resistance plate. Electricity is produced through the oscillating motion of the armature, with sinusoidaldistributed magnetic field, through a poly-phase coil stator. The connection between the armature and the resistance plate implies the use of a low-friction seal, which allows the rigid connecting rod to move freely through the bottom of the canister without water leaks. The DC3 (Fig. 3) is the resonant-drive system and is a multiple-spar buoy (or starspar). The LEG is housed in the permanently sealed central spar, which is surrounded by four satellite spars located at equal distances from the central spar every $90^{\circ}$. The satellite spars provide stability against roll as well as reduction of overall draft of the buoy. The magnetic armature is attached to an inertial mass suspended by a system of springs, which has a natural resonant period defined by the spring constant of the system. In addition, the buoy has its own natural resonant period based on its crosssectional area and draft. The coupled resonance of the entire system should be tuned to the expected sea-state peak spectral period to obtain the optimum heave motion of the buoy and therefore, maximize armature motion. A major benefit to this system is that there are no external moving parts or seals that could potentially leak, however, this being a resonant based system implies a narrow band response which decreases performance when the sea-state peak spectral period is not matched to that of the system. This 
requires that the system is tuned to match the peak spectral period that will be seen most often at a specific deployment site. This could be overcome to some degree through the use of active tuning of the system, through various electrical or mechanical controls, but is not part of the scope of this work.
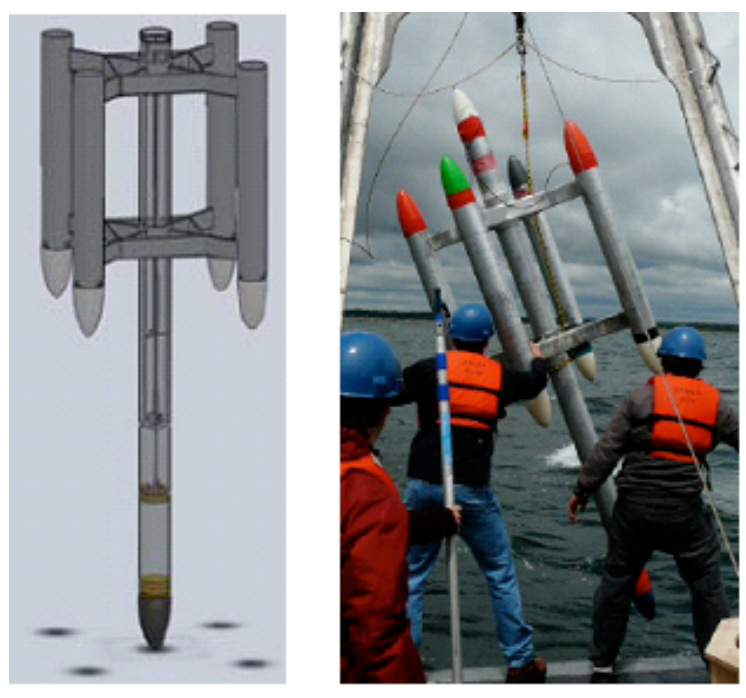

Figure 3. DC3 wave energy buoy; conceptual drawing (left) and 1:4 scale prototype (right)

\section{NUMERICAL MODEL}

Reference [6] provides a full summary of model development and preliminary dry testing of the LEG for initial model validation, of which the main points will be represented here. In order to either simulate or experimentally measure the expected performance of a LEG rigidly driven by the ocean wave surface elevation, a realistic model of the ocean waves and their kinematics is first established with the use of standard linear wave theory with harmonic superposition [5]. Equation (1) describes the ocean surface elevation $\eta$ at the systems location as the sum of $N_{w}$ sinusoidal wave components, each with amplitude $A_{i}$, circular frequency $\omega_{i}$, phase $\psi_{i}$ and wavenumber $k_{i}$. Each wavenumber satisfies the well-known linear dispersion relation given in (2), where $h$ is the ocean depth and $g$ is the local gravitational acceleration. Note, that $N_{w}=50$ is assumed in the following.

$$
\begin{gathered}
\eta(t)=\sum_{i=1}^{N_{w}} A_{i} \cdot \cos \left(k_{i} \cdot y+\omega_{i} \cdot t+\psi_{i}\right) \\
\omega_{i}^{2}=g \cdot k_{i} \cdot \tanh \left(k_{i} \cdot h\right)
\end{gathered}
$$

For this work, the sinusoidal component amplitudes are provided by a modified JONSWAP spectrum for developing seas. The JONSWAP spectral shape is a two parameter model in which both $A_{s}$ and $T_{p}$ are specified, and these parameters are not completely arbitrary, since they must take on values that are consistent with a realistic wind speed. Equation (3) shows the relation for wind speed in the case of JONSWAP spectra. Understanding of these relations is clearly important for ocean energy harvesting system design, since the values of the sea-state parameters $A_{s}$ and $T_{p}$ are not arbitrary in the ocean environment.

$$
u_{10}=\frac{\left(53 \cdot A_{s}\right)^{4}}{T_{p}{ }^{7}}
$$

For a rigid-drive system, standard linear wave theory and the use of the modified JONSWAP spectra allows for greatly simplified modeling and testing of the LEG performance, since the armature motion is moving in concert with the surface elevation. This leaves the electrical performance as the more critical aspect for modeling rigidly driven systems.

Electromagnetic field analysis provides a set of equations that capture the important voltage and current response of the LEG output. The model is a direct physical model (i.e. not an equivalent circuit model) with no equivalent parameters and no requirement for finite element analysis. Essentially, very accurate predictions are obtained for known geometrical, physical and material properties of the device. The only free parameter in the LEG model is a speed dependent power efficiency curve related to heating loss, which is experimentally calibrated. The efficiency has experimentally been found to be high for LEG's, in this application, due to the low armature speeds associated with the direct driving by ocean waves. Ignoring copper losses, which can be easily handled in the model, other losses which affect the LEG efficiency (e.g. hysteresis, eddy currents, etc) generally are no more than $10 \%$. Hence, a key finding in this work is that LEG analysis is simplified for the low power and the low speed ranges consistent with this application.

The electrical/mechanical interactions of the LEG, with the external driving forces from wave action, are clearly important to determine output power generation. Five LEG forces are considered: gravity, drive force, electromagnetic thrust, armature friction and spring force. This leads to additional state equations that are combined with the wave equations, buoy hydrodynamic equations and load model equations to form a complete system of equations. The LEG system creates two additional mechanical nonlinear state space equations. Thus, two more states and state equations are created and these are documented in the literature [5], [9], [10]. Electrical equations are also necessary, but do not necessarily require additional state variables in this formalism since generator inductance is usually negligible with low speeds.

The LEG model considers a cylindrical armature, much longer than the stator, made from permanent magnets. The magnets are fixed at equal spacing to generate an approximate sinusoidal distribution, in the longitudinal $z$ direction, for the radial component of magnetic flux density $B_{r}$. The radial component of magnetic flux density $B_{r}$ is approximated by a truncated Fourier series expansion with $N_{b}$ terms as shown in (4). Here, $C_{p p}$ is the number of magnet pairs per unit length and the series coefficients $b_{k}$ are dependent on radial distance. The reference frame moves with the armature, at velocity $v_{a}$ relative to the stator, in the longitudinal $z$-direction. 


$$
B_{r}(z)=\sum_{k=1}^{N_{B}} b_{k} \cdot \cos \left(2 \pi \cdot(2 \cdot k-1) \cdot C_{p p} \cdot z\right)
$$

The short length stator has coils that cover an integer number of magnet pairs $N_{c}$. The effective average diameter of the coil is $D_{\text {coil }}$. The number of coil turns for each slot, on a particular phase coil, is indicated as $N_{m n}$ with $n=1$ or 2 for each of the 2 phases and $m$ being a slot index.

The longitudinal coordinate $z_{m}$, for each slot in the moving reference frame, is given by (5), where $x_{a}$ is the armature displacement and $z_{s}$ is an arbitrary displacement offset that allows initial positioning of the stator at any point along the length of the armature.

$$
z_{m}(t)=z_{s}+x_{a}(t)+\frac{m-1}{4 \cdot C_{p p} \cdot N_{s}}
$$

The longitudinal force $F_{z}$ from both phases and all slots is given by (6), where the 2 phase currents in, for $n=1,2$ are orthogonal to the radial magnetic flux density $B_{r}$. Using the periodicity and symmetry allows simplification to the formula, as shown.

$$
F_{z}(t)=2 \cdot \pi \cdot D_{\text {coil }} \cdot N_{c} \cdot \sum_{n=1}^{2} \sum_{m=1}^{2 \cdot N_{s}} B_{r}\left(z=z_{m}(t)\right) \cdot N_{m n} \cdot i_{n}(t)
$$

Given that the present application is brute-force power harnessing by conversion to DC through a rectifier, the harmonic distortion of the generated $\mathrm{AC}$ output waveform is less important and there seems little benefit to have perfect sinusoidal distribution of the permanent magnet field or for the coil winding density. As a result, the LEG design and the analysis can be simplified by using the most basic coil winding method, which is to set $N_{s}=1, N_{11}=N_{22}$ and $N_{12}=N_{21}=0$. Even this simple arrangement can generate a reasonable approximate sinusoidal distribution with only one significant upper harmonic (i.e. $N_{b}=2$ ). It becomes necessary to calculate the effective magnetic field seen by a coil wound in a relatively wide slot; hence, many details are left out here. Since the application of Faraday's law is non-trivial in this geometry, determination of voltage is handled by considering power conservation, and equating mechanical power to electrical power and considering an equivalent speed dependent efficiency curve, which is determined experimentally. This works since efficiency is very high $(>90 \%)$ with the LEG designs and low speeds, presently.

The electrical model must be completed by specifying the load circuit conditions. For test purposes it is sometimes useful to use simple resistive loading which is straightforward to model. However, for practical implementation, a more sophisticated rectifying circuit is used. A DC/DC converter using an emulated resistance allows the power to be stored on batteries or super-capacitors for use by on-board sensors or communications modules. The circuit equations for the load circuit are straightforward to implement, and are included in the model. Fig. 4 shows a simplified schematic for the equivalent generator and load circuit. The generator is shown as an equivalent two phase source with phase resistance $R_{s}$ and phase inductance $L_{s}$. The load part of the circuit is accurate as a model, and is shown as a full-wave two-phase bridge configuration with an emulated load resistance, $R_{\text {emul }}$.

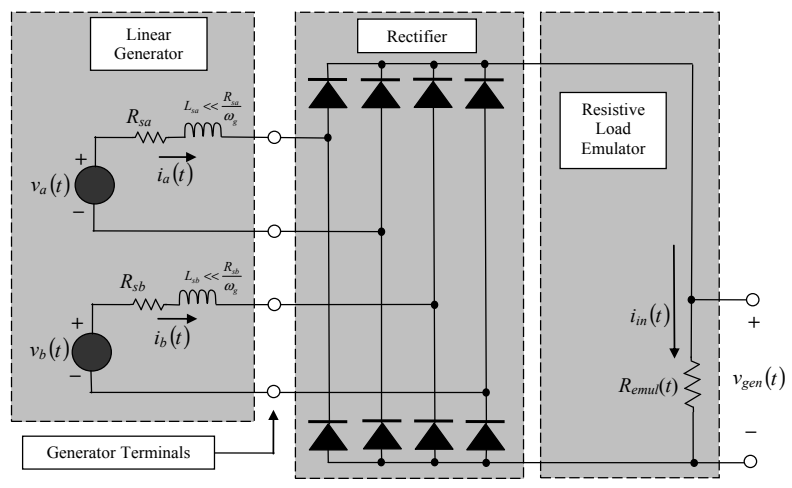

Figure 4. Equivalent circuit of generator and load

Simulation of practical directly driven and resonantly driven systems requires considerable extensions to the models discussed above in order to properly model. First, the buoys are not perfectly wave compliant and have their own response that must be accurately calculated. In the case of the direct-drive system, the resistance plate is not an unmovable object and also has its own response that must be quantified. In general, a simplified model with added mass and dissipation, but ignoring vortex shedding is used. Considerable effort has been made to accurately calculate the response of the buoys. The starting point is the usual equations of motion including all forces (spring force, generator force, hydrodynamic force, friction force, radiation force, etc), and buoy added mass. These force equations are established and discussed throughout the literature [6] and only the hydrodynamic force $F_{3}$ will be discussed here. A nonlinear correction is made for the buoy's added mass, at infinite frequency, which is assumed to be equal to one half of the instantaneous value of displaced water mass, with the sum of the individual spar added masses being used for the resonant drive system.

For hydrodynamic force $F_{3}$, the well-known WAMIT program is used to calculate the frequency dependent modulus $r_{3}$ and phase $\varphi_{3}$ of excitation force for the buoy under consideration. These parameters are typically used in a force formula, valid in the linear regime, and both modulus and phase depend on wave frequency, as well as on buoy shape, size and draft. The linear hydrodynamic force equation is modified with nonlinear corrections that account for large heave displacement, since buoy diameter is comparable to the interesting range of wave amplitude in practical cases. The main purpose of the nonlinear corrections to hydrodynamic force and added-mass are to prevent the simulations from displaying unrealistic behavior when occasional large heave excursions happen. Most of the time the buoy motion is in the linear excursion range, but with random waves, there are periodic large deviations which are then quickly damped. Equation (7) shows the formula for $F_{3}$ for the direct-drive system, where $\rho$ is water 
density, $x_{b}$ is buoy heave displacement and $D_{b}$ buoy diameter. Likewise, Equation (8) shows the formula for $F_{3}$ for the resonant-drive system, where $A_{i}$ is the amplitude of the $i^{\text {th }}$ wave component, $D_{s j}$ and $d_{s j}$ are the $j^{\text {th }}$ spar's diameter and draft, respectively.

$$
\begin{gathered}
F_{3}=\rho \cdot g \cdot\left(1-\left(\frac{2 \cdot x_{b}}{D_{b}}\right)^{2}\right) \cdot \sum_{i=1}^{N_{k i}}\left(1+k_{i} \cdot x_{b}\right) \cdot A_{i} \cdot r_{3}\left(\omega_{i}\right) \cdot \cos \left(\omega_{i} \cdot t+\phi_{3}\left(\omega_{i}\right)+\psi_{i}\right) \\
F_{3}(t)=\rho \cdot g \cdot \sum_{i=1}^{N_{w}} A_{i} \cdot \sum_{j=0}^{N_{s}} D_{s j}{ }^{3} \cdot \hat{r}_{3}\left(\frac{\omega_{i}}{\sqrt{D_{s j}}}\right) \cdot \cos \left(\omega_{i} \cdot t+\hat{\phi}_{3}\left(\frac{\omega_{i}}{\sqrt{D_{s j}}}\right)+\psi_{i}\right) \frac{\cosh \left(k_{i} \cdot\left(h-d_{s j}\right)\right)}{\cosh \left(k_{i} \cdot(h-10)\right)}
\end{gathered}
$$

\section{PRototype Testing}

Full scale buoy design is based on a targeted sea state of the 20 year average for Rhode Island shelf waters; with significant wave height $H_{s}=1.2 \mathrm{~m}$ and peak spectral period $T_{p}=4.5 \mathrm{~s}$. Prior to construction and testing of full scale prototypes, it is necessary to perform small scale testing in both laboratory settings and at-sea. Laboratory testing of 1:10 scale mini-prototypes of each system was first performed and described in [7]. The results of these tests, which compared favorably with numerical simulations, provided confidence in the design and allowed for 1:4 scale prototypes to be built and tested. The following sections provide brief descriptions of the 1:4 scale prototypes and field experiments in Narragansett Bay [8]. The experimental data was then compared to model simulation results for the purpose of model verification and validation. The model matches all buoy and generator parameters used in the field experiments and uses the JONSWAP spectrum determined from a waverider buoy as input to create a time series of sea surface elevation to represent the wave forcing seen during the field tests.

\section{A. Direct-Drive Buoy (DC2)}

Using Froude scaling, the 1:4 scale buoy is designed for a sea state of $H_{s}=0.3 \mathrm{~m}$ and $T_{p}=2.25 \mathrm{~s}$. The 1:4 scale DC2 consists of a $0.92 \mathrm{~m}$ diameter spherical float, rigidly attached to a $0.15 \mathrm{~m}$ diameter cylindrical canister which houses the LEG. The resistance plate has a diameter of $1.02 \mathrm{~m}$ a mass of $118.1 \mathrm{~kg}$. The total buoy mass is $208.1 \mathrm{~kg}$ to set the draft of the buoy such that the float is half submerged. In its static rest position, the buoy has a length of just over $3.5 \mathrm{~m}$ and the armature has a maximum displacement of $\pm 0.21 \mathrm{~m}$. Two field tests of the DC2 are described in [8]; the first in December 2011 and the second in May 2012. As described earlier, the LEG consists of a two-phase stator with a series of slots filled with copper windings through which a permanent magnet slider (armature) passes. The phase resistance of the stator used in the December test was $R_{s}=$ $1.6 \Omega$. For the May test, the LEG was upgraded with additional windings. The phase resistance of the stator for the May test was $R_{s}=4.8 \Omega$. The buoy is equipped with a 3axis accelerometer and gyroscope for measuring and recording buoy motion as well as two Hall Effect sensors for measuring the position of the permanent magnet armature relative to the buoy canister.
The wave conditions for the December test were measured using a waverider buoy designed and constructed by the Department of Ocean Engineering at URI. The waverider uses a 3-axis accelerometer and gyroscope to record accelerations and rates of rotation of the buoy. The measured buoy accelerations and angular velocities are converted to true accelerations relative to a non-rotating frame of reference and corrected for effects of buoy rotation. The heave acceleration is calculated by projecting measured accelerations to true vertical. A linear transfer function is then applied to the buoy acceleration spectrum to determine the wave spectrum. A JONSWAP wave spectrum is fit to the measured wave spectrum to provide the characteristic sea state parameters. This process is described in detail by [8]. The wave conditions for the period of usable data during the December test were equivalent to a JONSWAP spectrum with $H_{s}=0.63 \mathrm{~m}, T_{p}=3.33 \mathrm{~s}$ and peak enhancement factor $\gamma$ $=2$. The measured wave spectrum and JONSWAP spectrum are for this test is shown in Fig. 5. These conditions are significantly larger than the design conditions of $H_{s}=0.3 \mathrm{~m}$ and $T_{p}=2.25 \mathrm{~s}$. During the test, the LEG was loaded with a variable resistive load, which is represented by an equivalent (emulated) resistance $R_{\text {emul }}=10.75 \Omega$. Armature displacement ranged between $-0.15 \mathrm{~m}$ and $+0.20 \mathrm{~m}$ with armature velocity between $-0.5 \mathrm{~m} / \mathrm{s}$ and $+0.9 \mathrm{~m} / \mathrm{s}$. The RMS armature displacement $\left(z_{a}\right)$, armature velocity $\left(z_{a}^{*}\right)$ and harvested electrical power $(P)$ are given in Table I along with the results from numerical model simulations for the December test.

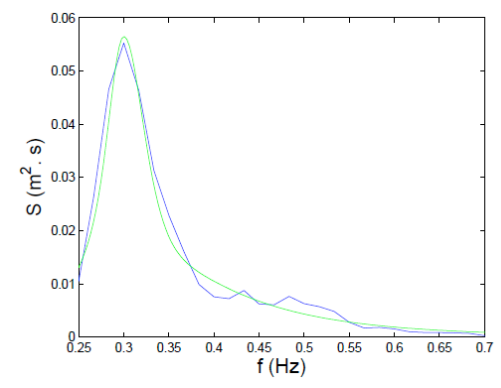

Figure 5. Wave spectrum measured using waverider (blue) and best-fit JONSWAP spectrum (green)

For the May test a commercially available waverider manufactured by Datawell BV of the Netherlands was utilized to measure the wave conditions. The Datawell waverider is rated for wave spectral periods between 1-30 s with an accuracy within $0.5 \%$ of measured values [11]. Approximately 15 minutes of data from the test, where the wave conditions were closest to the design wave conditions, was analyzed. The wave conditions were equivalent to a JONSWAP spectrum with $H_{s}=0.29 \mathrm{~m}$ and $T_{p}=2.5 \mathrm{~s}$ and $\gamma$ $=2.3$. The LEG was loaded with a fixed resistance $R_{L}=10$ $\Omega$ on each phase. Fig. 6 shows the armature displacement during the May test as well as the simulated armature displacement. The RMS armature displacement $\left(z_{a}\right)$, armature velocity $\left(z_{a}^{*}\right)$ and harvested electrical power $(P)$ are 
given in Table I along with the results from numerical model simulations for the May test.
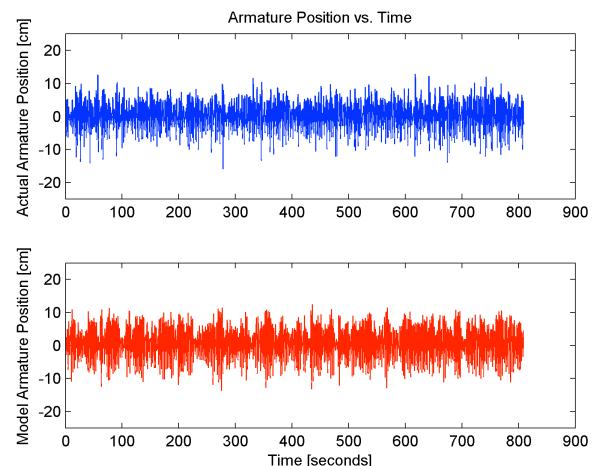

Figure 6. Armature displacement during May 2012 DC2 field test (top) and model simulation results (bottom)

The model simulations and experimental data appear to match very well for both the December and May field tests of the DC2. While RMS armature displacement is slightly greater for the simulations, the RMS velocity and mean harvested electrical power for the simulations and experiments are within $10 \%$.

TABLE I. COMPARISON OF EXPERIMENTAL AND MODEL RESUlTS

\begin{tabular}{|c|c|c|c|c|}
\hline \multirow{2}{*}{ Test Date (System) } & \multicolumn{4}{|c|}{ Table column subhead } \\
\cline { 2 - 5 } & Data & $\boldsymbol{z}_{\boldsymbol{a} \text { RMS }}$ & $\boldsymbol{z}_{\boldsymbol{a}}^{*}$ RMS & $\boldsymbol{P}_{\text {mean }}$ \\
\hline \multirow{2}{*}{ Dec. '11 (DC2) } & Experiment & $0.07 \mathrm{~m}$ & $0.21 \mathrm{~m} / \mathrm{s}$ & $0.42 \mathrm{~W}$ \\
\cline { 2 - 5 } & Model & $0.08 \mathrm{~m}$ & $0.19 \mathrm{~m} / \mathrm{s}$ & $0.40 \mathrm{~W}$ \\
\hline \multirow{2}{*}{ May '12 (DC2) } & Experiment & $0.04 \mathrm{~m}$ & $0.12 \mathrm{~m} / \mathrm{s}$ & $1.29 \mathrm{~W}$ \\
\cline { 2 - 5 } & Model & $0.05 \mathrm{~m}$ & $0.13 \mathrm{~m} / \mathrm{s}$ & $1.38 \mathrm{~W}$ \\
\hline \multirow{2}{*}{ June '12 (DC3) } & Experiment & $0.14 \mathrm{~m}$ & $0.40 \mathrm{~m} / \mathrm{s}$ & $0.41 \mathrm{~W}$ \\
\cline { 2 - 6 } & Model & $0.14 \mathrm{~m}$ & $0.39 \mathrm{~m} / \mathrm{s}$ & $0.44 \mathrm{~W}$ \\
\hline
\end{tabular}

\section{B. Resonant Buoy (DC3)}

Like the 1:4 scale DC2, the 1:4 scale DC3 buoy was designed for a sea state with $H_{s}=0.3 \mathrm{~m}$ and $T_{p}=2.25 \mathrm{~s}$. The central spar, which houses the LEG, consists of a $0.15 \mathrm{~m}$ diameter aluminum tube with an overall length of $3.5 \mathrm{~m}$, including nose cones. The four satellite spars use the same $0.15 \mathrm{~m}$ diameter tube with overall lengths of $1.6 \mathrm{~m}$ (including nose cones) and are placed such that the overall width of the buoy is $0.97 \mathrm{~m}$. The total mass of the buoy (including armature mass) is $104.6 \mathrm{~kg}$, which sets the draft of the central spar and satellite spars at $2.3 \mathrm{~m}$ and $0.8 \mathrm{~m}$, respectively. With an armature mass of $6.8 \mathrm{~kg}$ and armature spring constant of $102.5 \mathrm{~N} / \mathrm{m}$, the combined natural period of the buoy is $2.25 \mathrm{~s}$. The maximum armature displacement for the DC3 was $\pm 0.32 \mathrm{~m}$. Like the DC2, the DC3 is equipped with a 3-axis accelerometer and gyroscope for measuring and recording buoy motion. The phase resistance of the LEG for the June test was $R_{s}=9.8 \Omega$ and internal Hall Effect sensors measure position of the magnetic armature.
The field test of the DC3 was performed in June 2012, and as was the case with the May 2012 field test of the DC2, the Datawell waverider was used to measure wave conditions during the test. Approximately 25 minutes of data was collected and analyzed for the DC3 test, with wave conditions during the test being equivalent to a JONSWAP spectrum with $H_{s}=0.31 \mathrm{~m}, T_{p}=2.52 \mathrm{~s}$ and $\gamma=6$. Throughout the test, the LEG was loaded with an average emulated resistance $R_{\text {emul }}=8 \Omega$. The buoy heave acceleration, armature displacement $\left(z_{a}\right)$, armature velocity $\left(z_{a}\right)$ and harvested electrical power $(P)$ are shown in Fig. 7 through 10 along with the results from numerical model simulations for the DC3 field test. RMS armature displacement and velocity, as well as mean power are shown in Table I. The RMS buoy heave acceleration was $1.38 \mathrm{~m} / \mathrm{s}^{2}$ for the June test, compared with $1.44 \mathrm{~m} / \mathrm{s}^{2}$. Note the drift in armature position for the experimental results in Fig. 8, which is most likely due to low data decimation. It can be seen that the armature was using its full stroke range of $\pm 0.32 \mathrm{~m}$. Notice that the plots generally agree in magnitude, however do not line up exactly, which is due to using a manufactured time series from a JONSWAP spectrum instead of a time series with an exact replication of the sea surface during the test, which is not available.
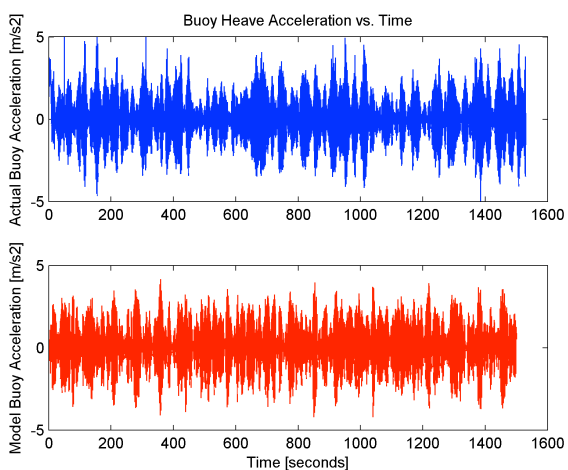

Figure 7. Buoy heave acceleration during June 2012 DC3 field test (top) and model simulation results (bottom)
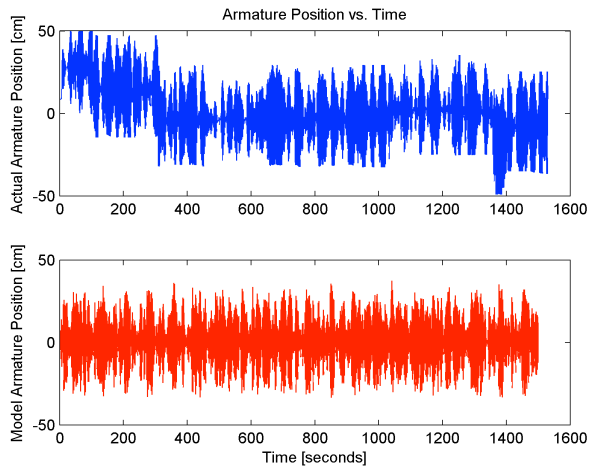

Figure 8. Armature displacement during June 2012 DC3 field test (top) and model simulation results (bottom) 

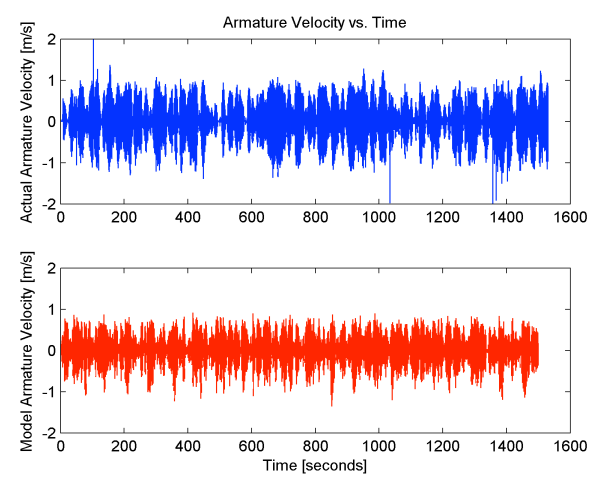

Figure 9. Armature velocity during June 2012 DC3 field test (top) and model simulation results (bottom)

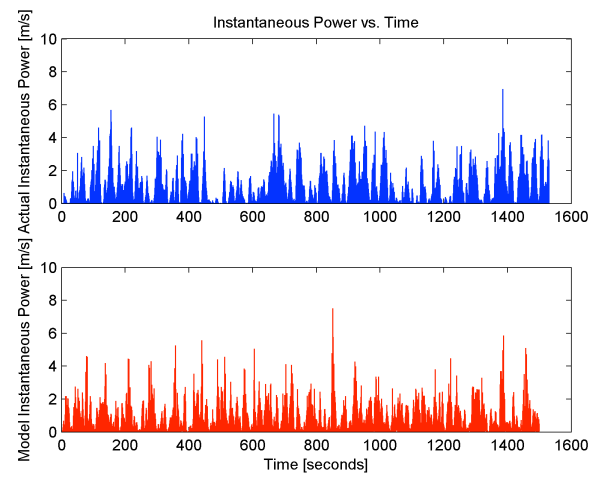

Figure 10. Instantaneous electrical power harvested during June 2012 DC3 field test (top) and model simulation results (bottom)

In the time since the June 2012 field test, the LEG for the DC3 has been replaced with a new generator more like the type used in the DC2. The new LEG stator has more windings and covers more magnet pairs of the slider than the previous DC2 LEG. The phase resistance of the new generator has increased to $R_{s}=12.7 \Omega$. In addition, the armature mass has been increased to provide more driving force though the stator to counteract the increase electromagnetic thrust and friction from the larger generator. New springs with the proper spring constant to obtain the correct design frequency were installed as well. A field test with the modified DC3 has not yet been performed, but will take place within the second half of 2013 . Preliminary modeling has shown that harvested electrical power is anticipated to be in the range of 5-10 W.

\section{CONCLUSION}

This paper presented a review of a wave-to-wire numerical model developed to analyze direct-drive and resonant-drive buoy systems for ocean wave energy harvesting. Field testing was completed with 1:4 scale prototype systems in Narragansett Bay and showed results that are in line with expected performance. The applications of interest have power requirements in the 1-10 W range. With generator improvements and scaling taken into consideration, this work has demonstrated the ability to achieve these requirements. In addition, the experimental data were used for verification and validation of the model. The results of the experiment were shown to match well with those from model simulations, with RMS armature displacement, RMS armature velocity and mean harvested electrical power generally falling within $10 \%$ of the measurements. This gives confidence in using model simulations to optimize the systems as well as providing assistance in the design of full scale wave energy harvesting systems.

\section{REFERENCES}

[1] K. Rhinefrank, E. B. Agamloh, A. von Jouanne, A. K. Wallace, J. Prudell, K. Kimble, J. Aills, E. Schmidt, P. Chan, B. Sweeny and A. Schacher, "Novel ocean energy permanent magnet linear generator buoy," Renewable Energy, vol. 31, no. 9, pp. 1279-1298, 2006.

[2] D. Elwood, S. C. Yim, J. Prudell, C. Stillinger, A. von Jouanne, T. Brekken, A. Brown, R. Paasch, "Design, construction, and Ocean testing of a taut-moored dual-body wave energy converter with a linear generator power take-off," Renewable Energy, vol. 35, no. 2, pp. 348-354, 2010.

[3] D. Elwood, A. Schacher, K. Rhinefrank, J. Prudell, S. Yim, E. Amon, T. Brekken, A. von Jouanne, "Numerical Modeling and Ocean Testing of a Direct-Drive Wave Energy Device Utilizing a Permanent Magnet Linear Generator for Power Take-Off," $28^{\text {th }}$ Int. Conf. on Ocean, Offshore and Arctic Eng., Honolulu, HI, USA, OMAE2009, vol. 4, pp. 817-824, 2009.

[4] M. Stalberg, R. Waters, O. Danielsson, and M. Leijon, "Influence of Generator Damping on Peak Power and Variance of Power for a Direct Drive Wave Energy Converter," Journal of offshore mechanics and Arctic engineering, ASME, vol. 130, no. 3, 2008.

[5] A. R. Grilli, J. Merrill, S. T. Grilli and M. L. Spaulding, Experimental and numerical study of spar buoy-magnet-spring oscillators used as energy absorbers," in Proc. $17^{\text {th }}$ Intl. Conf. Offshore and Polar Eng, No. 2007-JSC-569, 2007.

[6] S. P. Bastien, R. B. Sepe, Jr., A. R. Grilli, S. T. Grilli, and M. L. Spaulding, "Ocean Wave Energy Harvesting Buoy for Sensors," Proc. IEEE Energy Conversion Congress and Exposition, ECCE09, San Jose, CA, USA, pp. 3718-3725, 2009.

[7] S. T. Grilli, A. R. Grilli, S. P. Bastien, R. B. Sepe, Jr., and M. L. Spaulding, "Small Buoys for Energy Harvesting: Experimental and Numerical Modeling Studies," Proc. $21^{\text {st }}$ Int. Offshore and Polar Eng. Conf., ISOPE, Maui, HI, USA, pp. 598-605, 2011.

[8] J. Montgomery, Experimental and Numerical Study of a Wave Energy Harvesting Buoy, MS Thesis, Dept. of Ocean Engineering, University of Rhode Island, Kingston, 2012.

[9] A. Babarit, G. Duclos, A. H. Clement and J. C. Gilloteaux, "Latching control of a power take-off oscillator carried by a wave activated body," Proc. $20^{\text {th }}$ Int. Workshop on Waves \& Floating Bodies, pp. 1922, 2006.

[10] A. Babarit and A. H. Clement, "Optimal latching control of a wave energy device in regular and irregular waves," Appl. Ocean Res., 2006.

[11] Datawell BV, Waverider SG, http://www.datawell.nl , 2013. 